\title{
Collaborative Web-Based Simulation Platform for Construction Project Planning
}

\author{
Ali Ismail, Yaseen Srewil, and Raimar J. Scherer \\ Institute of Construction Informatics, \\ Dresden University of Technology, Dresden, Germany \\ \{Ali.Ismail, Yaseen.Srewil, Raimar.Scherer\}@tu-dresden.de
}

\begin{abstract}
This paper presents a process-based simulation toolkit CST and collaboration platform ProSIM to support planning of construction projects using simulation methods based on Building Information Modelling (BIM) and multi-model data exchange approaches. CST uses reference process models for construction activities and addresses data interoperability problems caused by the diversity of available CAD/BIM and project-planning software. The online collaboration platform connects design, planning, construction, and simulation experts together and enables an efficient communication and information sharing. This paper focus mainly on the collaboration and data interoperability aspects of CST and ProSIM in order to enable a wide adopting of simulation methods for planning of construction projects.
\end{abstract}

Keywords: project planning, simulation, data interoperability, collaboration.

\section{Introduction}

Simulation methods have been used to optimize and improve the planning quality in many industry sectors like manufacturing and logistic since long time, and they are integrated as an essential part of the whole design and planning process. However, in the construction industry, the use of simulation methods for planning has not been widely adopted, it is limited to analyse and solve problems in an ad-hoc manner (simulation study or simulation-on-demand) at certain time point of the project life.

Simulation studies have been carried out in most cases through external simulation service providers on project basis and they are not a basic part of planning process. The application of simulation techniques for construction projects is a very promising but also a challenging field of research [1]. While great advances have been made in construction simulation over the past few decades, adoption by industry has lagged, for three potential reasons: simulation is not accessible, it cannot handle the complexity of modern construction projects, and the benefits are not immediately obvious [2]. Creating reliable and reusable simulation models is very complex, combined with high costs of software licenses and personal training for in-house simulation. Thus, providing convenient simulation tools and web-based collaborative platforms with low-cost entry is crucial to promote a wider adoption of simulation in construction industry. 
This research work, which is partly developed in the project Mefisto "Management, Leadership, Information and Simulation in Construction" (http://www.mefisto-bau.de), aims to make a paradigm shifting of traditional simulation studies to be an integrated part of the planning process for construction projects. The main contributions in this research are: (1) Using formal reference process models based on BPMN to describe the construction and logistic activities, (2) Integration of a multi-model approach to exchange structured project data with the simulation environment to enhance data interoperability, (3) Implantation of a construction simulation toolkit CST and a collaboration platform ProSIM. CST is a process-based discrete event simulation toolkit for construction project planning [3]. It aims to accelerate the process of creating simulation models for production and logistic operations. Simulation models built with CST will help to verify the feasibility of a given schedule against a combination of different resource constraints or different building design alternatives. It aims to improve the quality of projects planning by reducing the total duration and cost of construction projects and improving the utilization rates of resources.

The target of this research is to promote a wide adoption of simulation methods to be an integrated part of the planning process for construction projects through the whole project life cycle. In this approach, simulation models will be created once during the early design phase and accompany the project progress from primary design and planning phases until the end of the construction work.

\section{Process-Based and Multi-Model Data Exchange Simulation Approach}

Construction is teamwork and successful team operations rely on collaboration. Because of the complexity of the construction industry, the multiple phases of the construction project lifecycle, the involvement of multidisciplinary teams (including owners, architects, consultants, engineers, contractors, sub-contractors, and suppliers), and the use of heterogeneous software and hardware systems/tools, systems integration becomes an important prerequisite to achieve efficient and effective collaboration [4].

The complexity of simulation models for construction projects and the increasing volume of information raise the need to adopt new collaboration and project data exchange methods. As the project size grows, the number and size of teams will increase. An interoperable and smooth data flow between planning and simulation experts and their tools is a key factor to reduce the time and efforts required to collect and update project data regularly. In our approach we use reference process models to manage the knowledge in the construction domain collaboratively and a new method to exchange project data based on multi-model method. 


\subsection{Reference Process Models}

Reference models are generic conceptual models that formalise recommended practices for a certain domain [5]. As the knowledge in every domain is keep evolving, the reference models will go through the known approach "discover, model, evaluate, and optimize" so new models will be added or existing models will be improved and detailed with time (Fig.1). Therefore, a continuous collaboration between planning, construction management and simulation teams is very important to validate and integrate the feedbacks during the construction phase and embedded them correctly in the reference models. The reference process modelling method allows identifying, capturing, documenting, improving and sharing knowledge about the best practice of construction processes and their related information

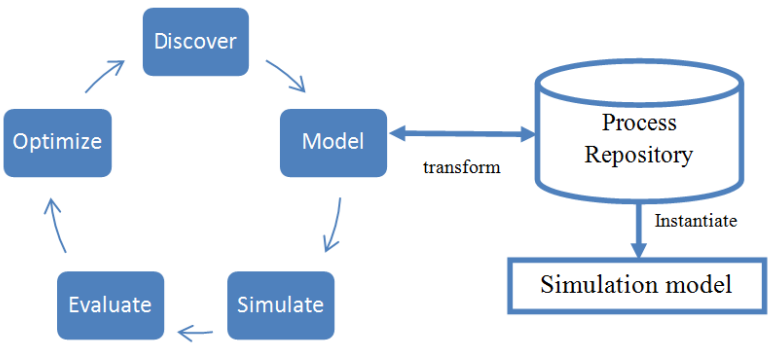

Fig. 1. Reference process models life cycle

In our approach, there is a clear separation between the core simulation engine and the process models for the application domains. Business Process Modelling and Notation (BPMN) models are used to capture and describe the logic of production and logistic operations and to transform them directly into simulation models.

Extension elements are introduced in order to define resource requirements and durations of tasks. Each activity or task inside the project schedule can be linked with a reference process model during simulation. In this way, the process models define the logic and level of details of simulation models. One needs to carefully examine every detail of the construction process and identify the major events and processes that will be presented in the simulation model in order to create a reliable simulation model [6]. The graphical representation of BPMN models makes models easy to understand and the formal specifications in XML allows transforming process models into simulation models automatically. The BPMN reference process models can be imported into a process repository in the simulation mode (Fig.2). The process repository includes reference process models for the best practices of various construction processes that are reusable across different construction projects and their data definitions of resources and productivity factors.

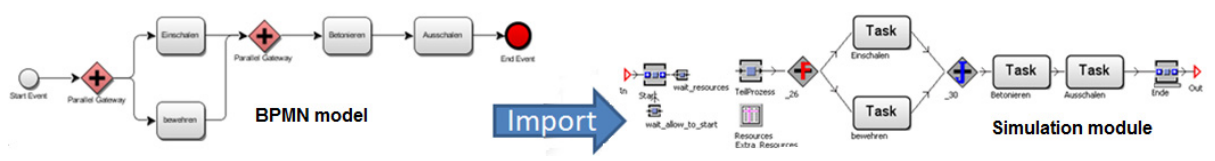

Fig. 2. Transformation of reference process models to simulation modules 


\subsection{Multi-Model Approach to Exchange Projects Data}

Multi-model project planning is a paradigm shift from the building-centric approach, which tries to add and link all project data with building models toward an equivalent interlinked data models using special link models. The multi-model method offers solutions to structural problems of $\mathrm{nD}$ modelling in construction information processes [7]. A multi-model container comprises several data files and includes, besides the meta-information about the container content, several application models as well as link models [8]. It allows the combination of heterogeneous application models from different domains and various data formats. Inside multi-model containers link models bind the application models together. The link models specify the relationships between items from different data models. Multi-model containers can be used to exchange associated models among the project stakeholders by a common format. In their entirety the multi-models on a construction project open up a multi-dimensional information space of interdependent application models that can be independently processed by the project participants. Each participant has the opportunity to produce new application models on his/her own responsibility and interlink them with existing models. Depending on the situation these newly created multi-models can be maintained locally or published project-wide as a basis for further planning and controlling tasks. In comparison to the often pursued integration of project information in central project databases or product model servers, this approach distributed model-based collaboration represents a paradigm shift [9]. Multi-model approach is integrated with CST through providing import/export interfaces.

\section{Collaboration Platform ProSIM}

ProSIM is a web application designed and implemented especially for CST to work as a communication and collaboration layer between the simulation team and other design and planning teams. The main functions provided by ProSIM are:

- Share project data and keep them up-to-date: BIM models, project schedules, process templates, productivity factors can be updated at any time to reflect the actual state of the available information.

- Import and view the simulation inputs of various data models and multimodel containers directly inside the web browser.

- View and evaluate simulation results through online viewers to display results inside a web browser or download them using standard formats

- Manage construction process templates.

- Manage productivity factors, definition of resource requirements, and task duration of construction activities.

- Add, edit and run simulation experiments remotely.

Simulation models and their inputs and results will be available and re-runnable anywhere and responsive to any data update. The visibility of all input data and 
simulation parameters helps to identify errors and leads at the end to a better understanding among the planning and simulation teams and offers a high level of transparency and trust. Available API interfaces beside the simulation database connectivity make it possible to build integrated data management services around the simulation core components and offer it as Software as a service (SaaS).

Web-based simulation has many benefits over the classical desktop simulation systems, main advantages of using a web-based simulation tools are [10]:

(a) Collaboration: Communication and interaction are one of the essential factors to achieve a successful simulation project, (b) Cost reduction: traditionally the starting investment for a typical simulation environment or external simulation consultant are on a high level, the web-based interface allows new business models for the use of simulation services, which can be rented for an interval of time, this resulting in savings in terms of possible previous prohibitive factors of time and cost, (c) Wide availability: A Web-based simulation application can be used from anywhere in the world with an Internet connection, (d) Integration and interoperability: A Web-based tool can integrate and interoperate with both existing and future Web-based applications.

The main ProSIM collaboration features in the different project phases are:

\section{Pre Planning phase}

- Share and manage information about reference process models, productivity factors for workers and machines, and resource definitions of construction activities.

- Define the minimal requirements of information in BIM models and modelling guidelines based on the reference process models and scope of simulation study.

\section{Design and planning phase:}

- Provide import interfaces and online viewers for BIM and project schedules.

- Visualize and archive results for each simulation scenario.

- Change simulation parameters and run simulation scenarios remotely.

\section{Construction phase:}

- Collect real-time data from construction site and update the simulation model according to the real project progress and the changes in the construction site layout.

- Update estimated productivity factors and duration formulas based on actual duration of executed tasks.

- Improve reference process models continuously.

The real-time data collection aims to support project planning and simulation with accurate feedback during project execution phase. For this purpose, a developed solution based on RFID technology is envisioned [11]. This solution proposed a mechanism for semi-automation data acquisition on construction site throughout coupling the BIM/planning models and the physical construction using cheaper passive RFID tags. From collected and validated data the construction process progress can be derived and therefore as-built data extracted. This information can be send as inputs data to the ProSIM platform using an API interface leading to short-term simulation loops. 


\section{Prototype Implementation and Validation}

This section describes briefly the system architecture and the prototype implementation of ProSIM.

\subsection{Platform Architecture}

The platform architecture consists of five main modules (Fig. 3): (1) Simulation engine (2) Web server (3) Simulation database (4) BIM data server (5) ProSIM web application.

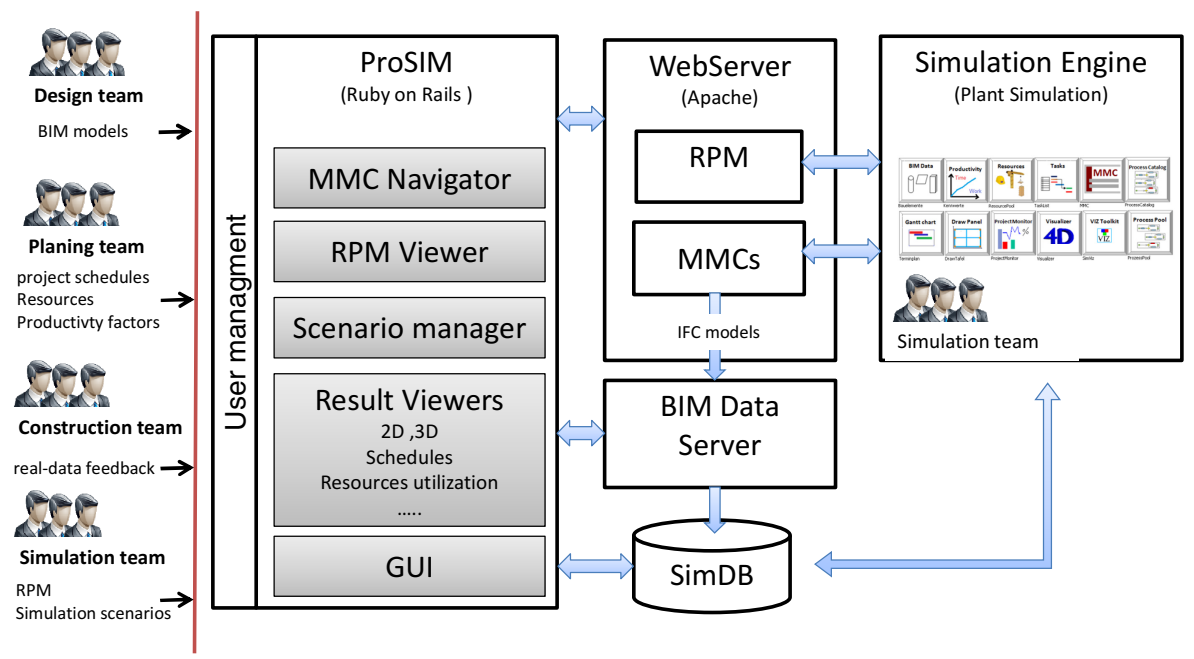

Fig. 3. System architecture and data flow

\subsection{ProSIM; Technical Implementation}

The ProSIM platform is designed as a Web-based system and implemented using the framework Ruby on Rails and the Web application is deployed via the Apache web server. The server-side data are stored in a SQLite database. The BIM and 4D simulation results models can be shown using a WebGL viewer based on the JavaScript 3D library "threejs" and Collada format. Ruby on Rails is suitable for rapid development; it emphasizes the use of well-known software engineering patterns and principles, such as active record pattern, and model-view-controller. The technical implementation has resulted in a rich web application with a REST API interface. 


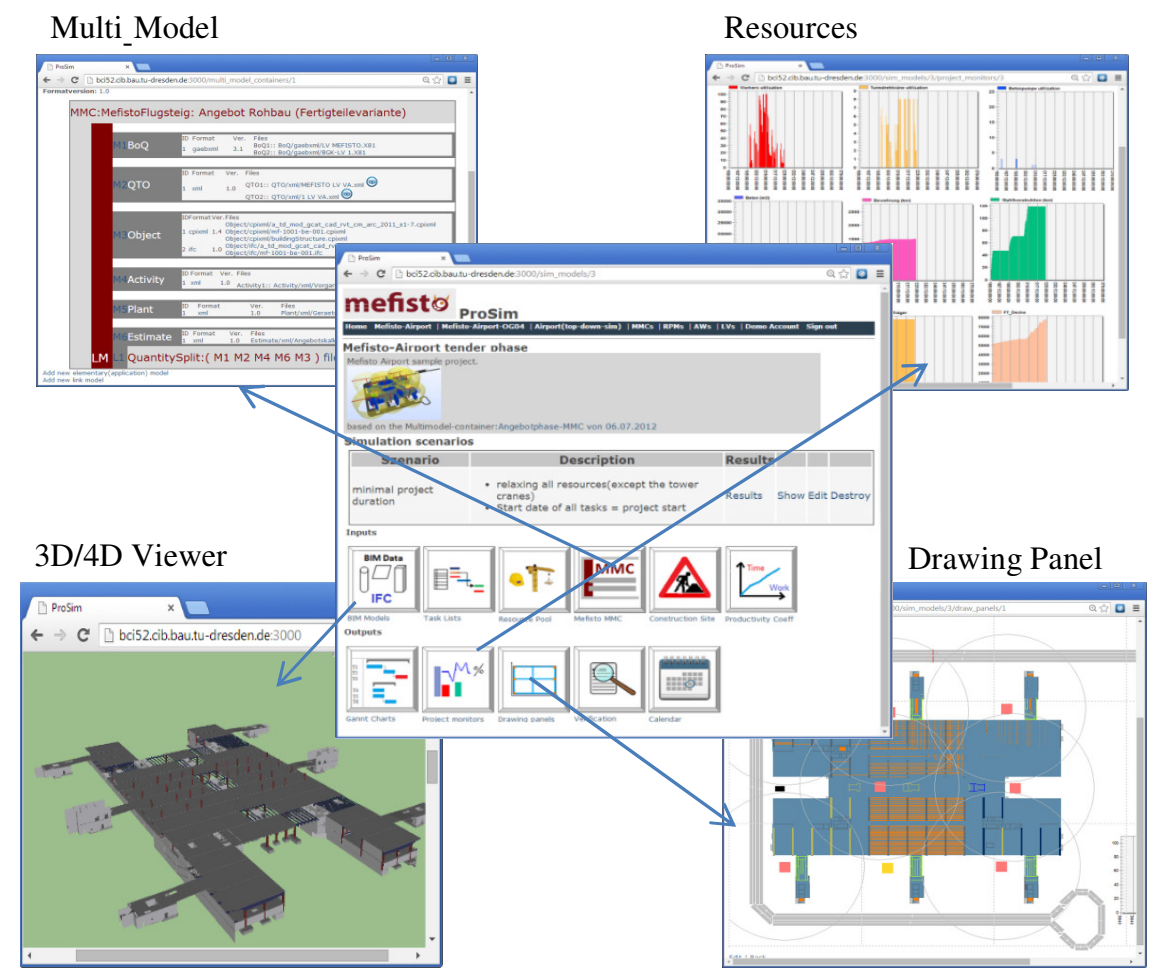

Fig. 4. A screenshots from a test case simulation model inside ProSIM

\subsection{Validation and Test Cases}

The CST simulation toolkit and the prototype of ProSIM platform were tested and validated based on two construction projects (multi-storey office building and an airport terminal). The easy access of projects data and simulation models through the online platform encouraged and enabled a collaborative work between different planning and simulation experts from different firms and helped to verify all input parameters and simulation results effectively. Some snapshots of a test case simulation model inside ProSIM are shown in Fig. 4. Simulation models of test cases can be accessed at: http://bci52.cib.bau.tu-dresden.de:3000

\section{Conclusions}

The target of this research is to promote a wide adoption of simulation methods to support construction project planning. Systems integration and collaboration are believed to be the key enabling technologies that drive the construction industry in improving productivity and efficiency [4]. This paper presented the integration between a process-based simulation toolkit and a collaborative platform. It discussed 
also the use of reference process models to capture and manage knowledge in construction domain and the use of multi-models improve data interoperability.

Acknowledgments. This research in this paper was enabled by the financial support of the German Federal Ministry of Education and Research (BMBF), Department of ICT under contract $\mathrm{n}^{\circ}$ 01IA09001A, which is herewith gratefully acknowledged.

\section{References}

1. Lucko, G., Benjamin, P.C., Madden, M.G.: Harnessing the power of simulation in the project management/decision support aspects of the construction industry. In: Proceedings of the Winter Simulation Conference (2008)

2. AbouRizk, S.: A Construction Synthetic Environment Integrating Visualization and Simulation ConVR 2011, p. 11 (2011)

3. Ismail, A., Scherer, R.: Process-based simulation library for construction project planning. In: Jain, S., Creasey, R.R., Himmelspach, J., White, K.P., Fu, M. (eds.) Proceedings of the 2011 Winter Simulation Conference (2011)

4. Shen, W., Hao, Q., Helium, M., Neelamkavil, J., Xie, H., Dickinson, J., Thomas, R., Pardasani, A., Xue, H.: Systems integration and collaboration in architecture, engineering, construction, and facilities management: A review. Enabling Technologies for Collaborative Design, Advanced Engineering Informatics 24(2), 196-207 (2010)

5. Rosemann, M.: Application reference models and building blocks for management and control (ERP Systems). In: Bernus, P., Nemes, L., Schmidt, G. (eds.) Handbook of Enterprise Architecture, pp. 595-615. Springer, Berlin (2002)

6. Aouad, G., Lee, A., Wu, S.: nD modelling for collaborative working in construction. Architectural Engineering and Design Management (1), 33-44 (2006)

7. Akhavian, R., Behzadan, H.A.: Dynamic Simulation of Construction Activities Using Real Time Field Data Collection. In: European Group for Intelligent Computing in Engineering Workshop (2011)

8. Schapke, S.-E., Scherer, R.J.: A distributed multi-model based Management Information System for simulation and decision making on construction projects. Submitted to Advanced Engineering Informatics Journal, Special Issues of the ICCCBE \& EG-ICE10 Conference (2010)

9. Schapke, S.-E., Pflug, C.: Multi-models: New potentials for the combined use of planning and controlling information. Transparent - das Magazin 37 (June 2012)

10. Byrne, J., Heavey, C., Byrne, P.J.: A review of Web-based simulation and supporting tools. Simulation Modelling Practice and Theory 18, 253-276 (2010)

11. Srewil, Y., Scherer, R.J.: Effective construction process monitoring and control through a collaborative Cyber-Physical approach. In: Camarinha-Matos, L.M., Scherer, R.J. (eds.) PRO-VE 2013. IFIP AICT, vol. 408, pp. 172-179. Springer, Heidelberg (2013) 